\title{
Comparison Results for Garch Processes
}

\author{
Fabio Bellini * Franco Pellerey †, Carlo Sgarra ${ }^{\ddagger}$ and Salimeh Yasaei Sekeh §
}

November 8, 2018

\begin{abstract}
We consider the problem of stochastic comparison of general Garchlike processes, for different parameters and different distributions of the innovations. We identify several stochastic orders that are propagated from the innovations to the Garch process itself, and discuss their interpretations. We focus on the convex order and show that in the case of symmetric innovations it is also propagated to the cumulated sums of the Garch process. More generally, we discuss multivariate comparison results related to the multivariate convex and supermodular order. Finally we discuss ordering with respect to the parameters in the Garch $(1,1)$ case. Keywords: Garch, Convex Order, Peakedness, Kurtosis, Supermodularity.
\end{abstract}

\section{Introduction}

An extensive literature is available on applications of stochastic orders to finance and insurance markets. The implications of stochastic orders for derivative pricing and risk management are relevant. The increasing dependence of european option prices by the riskiness of the underlying it is a well known property for basic models like that of Black-Merton-Scholes, in which riskiness is expressed in terms of the logreturns distribution variance: the uncertainty is quantified there through the dispersion around the expected value and the distribution functions can be ordered according to their "peakedness"; the larger is the dispersions, the higher the option prices. This very elementary and intuitive observation for simple models become more involved when turning attention to more complex models, where a more rigorous approach is necessary in order to avoid wrong conclusions.

The Black-Merton-Scholes model is nowadays considered fairly inadequate to describe the asset price dynamics; several empirical facts cannot be explained on

\footnotetext{
${ }^{*}$ Dipartimento di Metodi Quantitativi, Universitá di Milano Bicocca, Italy. E-mail: fabio.bellini@unimib.it

${ }^{\dagger}$ Dipartimento di Matematica, Politecnico di Torino, Italy. E-mail: franco.pellerey@polito.it

‡Dipartimento di Matematica, Politecnico di Milano, Italy. E-mail: carlo.sgarra@polimi.it

$\S$ Department of Statistics, Ferdowsi University of Mashhad, Iran. E-mail: sayasaei@yahoo.com
} 
the basis of this model: some statistical features exhibited by logreturns like fat tails, volatility clustering, aggregational Gaussianity and the so-called leverage effect are completely outside of the prevision properties of the Black-MertonScholes model. Moreover a very relevant phenomenon exhibited by option prices, the "volatility smile" (and its term structure) cannot be explained on this model basis. In order to provide a more satisfactory description several different models have been introduced. Some of these models introduce a stocastic dependence in volatility and/or jumps in asset logreturns (and/or in volatility) dynamics both in continuous and discrete time setting.

Among discrete time models introduced in order to remove some of the Black-Merton-Scholes model drawbacks, the class of Autoregressive Conditioned Heteroschedastic $(\mathrm{ARCH})$ models introduced by Engle [6] and their general extension (Garch models) proposed by Bollerslev in 4 have risen considerable interest.

Several results related to stochastic orders are available for the continuous time models class: in 2, where a systematic investigation on semimartingale models is performed; the models considered there include the Heston and the Barndorff-Nielsen and Shephard models. In [1] T. Møller provides some results on stochastic orders in a dynamic reinsurance market where the traded risk process is driven by a compound Poisson process and the claim amount is unbounded. Stochastic order properties have been used to obtain bounds for option prices in incomplete markets; the literature focused on this subject is quite extensive and we just mention the papers by El Karoui et al. 5, by Bellamy and Jeanblanc, and by Gushchin and Mordecki 8 .

The purpose of the present paper is to present a systematic investigation of stochastic orders propagation in a Garch context.

Comparison with stochastic orders in incomplete market models can give rise to different classes of problems: first can be considered the comparison of models under the same probability measure but with different parametric specification, second it can be examined the problem of comparing the same model under different probability measures; as a matter of fact, when markets are incomplete, there are several probability measures equivalent to the historical one, under which the dynamics of prices can be given. In this paper we shall focus on the first class of problems mentioned: we shall provide a systematic comparison of logreturns and then of prices when the model parameters change, but the dynamics is specified under the same probability measure. In a Garch context the parameters entering into play are three parameters assuming a numerical value and the innovations, which are random IID variables for which the density function is assigned. We just mentioned that stochastic order results have important implications on option pricing and this holds true also in a Garch context: in particular, convex order relations on logreturn sums imply increasing convex order relations on the underlying price, hence on european call option prices and this can be considered the main relevance of our results from an application viewpoint.

We like to present a numerical illustration in order to motivate our investigation. Fig 1 compare the densities of the logreturn sums in a Garch $(1,1)$ model 


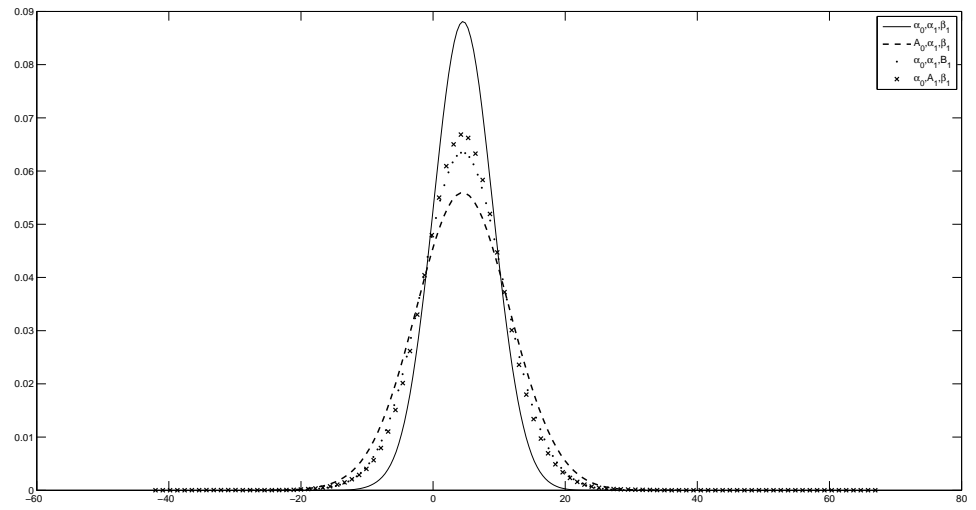

Figure 1: Comparison of Logreturn Sum Densities

with respect to variations in the parameters $\alpha_{0}, \alpha_{1}, \beta_{1}$; in the continuous line all parameters assume the value 0.2 , while the other lines represent the same density, but with parameters $\alpha_{0}=0.5, \alpha_{1}=0.5$ and $\beta_{1}=0.5$ respectively (the parameter assuming the value 0.5 in indicated by a capital letter in each curve caption). The sums include the first 50 terms of the logreturn sequence.

The innovations, assumed to be independent and identically distributed, and the initial variance, are chosen as standard Gaussian random variables. It is evident how the usual real numbers order relation between parameters implies some ordering on logreturn sums; it then arises as a natural question to ask if this simple remark can be made rigorous and if this conclusion can be cast into a more general framework considering different kind of stochastic orders and comparing stochastic order propagation from innovations to logreturns and to logreturns sums.

In the following section we present briefly the Garch models and an auxiliary lemma. In Section 3 we present the univariate stochastic comparisons for logreturns in a Garch setting. In Section 4 we provide results on some related orders, while in Section 5 the convex order propagation of the logreturn sums is investigated together with its implications on asset and european call option prices, while their multivariate convex order propagation is the subject of Section 6 . We focus our attention on the Garch $(1,1)$ case in the last section.

The main results provided in this paper require the assumption of a symmetric probability density for innovations. The extension of the stochastic order propagation analysis presented here to the case of non-symmetric innovation densities would be of great interest: both the convex order propagation result and the comparison with respect to parameter variations will be the subject of future investigation in this more general setting. Moreover the identification of some convex multivariate order which naturally propagate from innovations 
to logreturns is another target on which our research interest will be focused. These will be the subject of our future work.

\section{General Garch models}

We consider Garch models of two different very general forms; the first model (M1) is:

$$
\left\{\begin{array}{c}
X_{n}=\sigma_{n} \varepsilon_{n}, n=0,1, . . \\
\varepsilon_{n} \perp \sigma_{n}, E\left[\varepsilon_{n}\right]=0 \\
\sigma_{n+1}=f^{I}\left(\left|\varepsilon_{n}\right|, \sigma_{n}\right)
\end{array}\right.
$$

with $f^{I}: \mathbb{R}_{+}^{2} \rightarrow \mathbb{R}_{+}$increasing and componentwise convex (ccx for brevity).

The second model (M2) is

$$
\left\{\begin{array}{c}
X_{n}=\sigma_{n} \varepsilon_{n}, n=0,1, . . \\
\varepsilon_{n} \perp \sigma_{n}, E\left[\varepsilon_{n}\right]=0 \\
\sigma_{n+1}^{2}=f^{I I}\left(\varepsilon_{n}^{2}, \sigma_{n}^{2}\right)
\end{array}\right.
$$

with $f^{I I}: \mathbb{R}_{+}^{2} \rightarrow \mathbb{R}_{+}$increasing and ccx. In both cases the innovations $\varepsilon_{n}$ are independent and identically distributed (i.i.d.). When discussing the propagation of variability orderings, the normalization requirement $E\left[\varepsilon_{n}^{2}\right]=1$ will be dropped. The difference between model M1 and model M2 is that in the first case the recursive dynamics is defined in terms of the volatility $\sigma_{n}$, while in the second it is defined in terms of the variance $\sigma_{n}^{2}$.

The usual Garch $(1,1)$ model is a particular case of both M1 and M2, and is defined as follows:

$$
\left\{\begin{array}{c}
X_{n}=\sigma_{n} \varepsilon_{n}, n=0,1, . . \\
\sigma_{n+1}^{2}=\alpha_{0}+\alpha_{1} X_{n}^{2}+\beta_{1} \sigma_{n}^{2}
\end{array}\right.
$$

with $\alpha_{0}, \alpha_{1}, \beta_{1}>0$ and $\alpha_{1}+\beta_{1}<1$, in order to guarantee covariance station-

arity. Both models start with a possibly random $\sigma_{0}>0$, by drawing a random $\varepsilon_{0}$.

The recursive equations for $\sigma_{n+1}$ and $\sigma_{n+1}^{2}$ are examples of "stochastic recurrences" in the sense of Chapter 4 of [10]. For the explicit expression of their solutions, we introduce the following notations:

$$
\begin{aligned}
\sigma_{n+1} & :=g_{n+1}^{I}\left(\sigma_{0},\left|\varepsilon_{0}\right|, \ldots,\left|\varepsilon_{n}\right|\right) \\
\sigma_{n+1}^{2} & :=g_{n+1}^{I I}\left(\sigma_{0}^{2}, \varepsilon_{0}^{2}, \ldots, \varepsilon_{n}^{2}\right)
\end{aligned}
$$

As in [10, we have the following:

Lemma 1 Let $g_{n+1}^{I}, g_{n+1}^{I I}: \mathbb{R}_{+}^{n+2} \rightarrow \mathbb{R}_{+}$be defined as in (4). Then $g_{n+1}^{I}$ and $g_{n+1}^{I I}$ are increasing and componentwise convex.

Proof. We have clearly $g_{n+1}^{I}\left(\sigma_{0},\left|\varepsilon_{0}\right|, \ldots,\left|\varepsilon_{n}\right|\right)=f^{I}\left(\left|\varepsilon_{n}\right|, \ldots, f^{I}\left(\left|\varepsilon_{0}\right|, \sigma_{0}\right)\right)$ and $g_{n+1}^{I I}\left(\sigma_{0}^{2}, \varepsilon_{0}^{2}, \ldots, \varepsilon_{n}^{2}\right)=f^{I I}\left(\varepsilon_{n}^{2}, \ldots, f^{I I}\left(\varepsilon_{0}^{2}, \sigma_{0}^{2}\right)\right)$; since $f^{I}$ and $f^{I I}$ are increasing and ccx, then also their compositions in (4) are increasing and ccx (see Meester and Shanthikumar [9] for this last assertion and further properties of increasing and ccx functions). 


\section{Univariate comparisons of $X_{n}$}

The aim of this section is to establish comparison results for $X_{n}$ when the distributions of the innovations are changed from $\varepsilon_{k}$ to $\widetilde{\varepsilon}_{k}$. In order to establish these results, the assumption that the innovations are identically distributed is not necessary (while the independency assumption is essential). In the following theorems only the distribution of a single innovation $\varepsilon_{k}$ will be changed, and the impact of this change on $X_{n}$ will be investigated.

We recall the definitions of the basic stochastic orderings:

Definition 2 The random variable $X$ is said to be smaller than $Y$ in the usual stochastic order [convex order, increasing convex order], denoted by $X \leq{ }_{s t} Y$ $\left[X \leq_{c x} Y, X \leq_{i c x} Y\right]$, if $E[\phi(X)] \leq E[\phi(Y)]$ for all increasing [convex, increasing convex] functions $\phi: \mathbb{R} \rightarrow \mathbb{R}$ for which the expectations exist.

We will see that in the general context of models M1 and M2 the orderings that are naturally propagated from the innovations $\varepsilon_{k}$ to $X_{n}$ are the $\leq_{s t}$ and the $\leq_{i c x}$ ordering between absolute values or squared variables. This clearly completely modifies their interpretation; in particular, in the next section we will see that the $\leq_{s t}$ ordering between absolute values or squares can be interpreted as a variability ordering, while the $\leq_{i c x}$ ordering between absolute values or squares can be interpreted as a kurtosis ordering.

In order to establish these results, we proceed in two steps: first we consider the volatilities $\sigma_{n}$ and then the variables $X_{n}$. The first step is an immediate consequence of Lemma 1 .

Theorem 3 Comparisons of $\sigma_{n}$ and $\sigma_{n}^{2}$

a) Let $\sigma_{n+1}$ be as in (1) and $\left|\varepsilon_{k}\right| \preceq_{s t}\left|\widetilde{\varepsilon}_{k}\right|$; it follows that $\sigma_{n+1} \preceq_{s t} \widetilde{\sigma}_{n+1}$.

b) Let $\sigma_{n+1}$ be as in (1) and $\left|\varepsilon_{k}\right| \preceq_{i c x}\left|\widetilde{\varepsilon}_{k}\right|$; it follows that $\sigma_{n+1} \preceq_{i c x} \widetilde{\sigma}_{n+1}$.

c) Let $\sigma_{n+1}^{2}$ be as in (2) and $\varepsilon_{k}^{2} \preceq$ st $\widetilde{\varepsilon}_{k}^{2}$; it follows that $\sigma_{n+1}^{2} \preceq_{s t} \widetilde{\sigma}_{n+1}^{2}$.

d) Let $\sigma_{n+1}^{2}$ be as in (2) and $\varepsilon_{k}^{2} \preceq_{i c x} \widetilde{\varepsilon}_{k}^{2}$; it follows that $\sigma_{n+1}^{2} \preceq_{i c x} \widetilde{\sigma}_{n+1}^{2}$.

Proof. Since from Lemma 1 in model M1 $\sigma_{n+1}=g_{n+1}^{I}\left(\sigma_{0},\left|\varepsilon_{0}\right|, \ldots,\left|\varepsilon_{n}\right|\right)$ with $g_{n+1}^{I}$ increasing and ccx, item a) and b) follows respectively fromTheorem 1.A.3 in 13 and Theorem 4.A.15 in 13 . Similarly, since from Lemma 1 in model M2 $\sigma_{n+1}^{2}=g_{n+1}^{I I}\left(\sigma_{0}^{2}, \varepsilon_{0}^{2}, \ldots, \varepsilon_{n}^{2}\right)$ with $g_{n+1}^{I I}$ increasing and ccx, from the same theorems we get c) and $\mathrm{d}$ ).

The comparison results for $\sigma_{n}$ and $\sigma_{n}^{2}$ lead to the following comparisons of the variables $X_{n}$ :

Theorem 4 Comparisons of $X_{n}$

a) Let $X_{n}$ be as in (11) and $\left|\varepsilon_{k}\right| \preceq_{s t}\left|\widetilde{\varepsilon}_{k}\right|$; it follows that $\left|X_{n}\right| \preceq_{s t}\left|\widetilde{X}_{n}\right|$.

b) Let $X_{n}$ be as in (11) and $\left|\varepsilon_{k}\right| \preceq_{i c x}\left|\widetilde{\varepsilon}_{k}\right|$; it follows that $\left|X_{n}\right| \preceq_{i c x}\left|\widetilde{X}_{n}\right|$.

c) Let $X_{n}$ be as in (2) and $\varepsilon_{k}^{2} \preceq_{s t} \widetilde{\varepsilon}_{k}^{2}$; it follows that $X_{n}^{2} \preceq_{s t} \widetilde{X}_{n}^{2}$.

d) Let $X_{n}$ be as in (2) and $\varepsilon_{k}^{2} \preceq_{i c x} \widetilde{\varepsilon}_{k}^{2}$; it follows that $X_{n}^{2} \preceq_{i c x} \widetilde{X}_{n}^{2}$. 
Proof. Since $\left|X_{n}\right|=\sigma_{n}\left|\varepsilon_{n}\right|$ and $X_{n}^{2}=\sigma_{n}^{2} \varepsilon_{n}^{2}$, with $\sigma_{n}$ independent from $\varepsilon_{n}$, items a), and c) follow from Theorem 1.A.3 (b) in [13. Similarly item b) and d) follow from Theorem 4.A.15 in [13].

A natural question that arises at this point is if also the convex order is propagated, that is if $\varepsilon_{k} \preceq_{c x} \widetilde{\varepsilon}_{k} \Rightarrow X_{n} \preceq_{c x} \widetilde{X}_{n}$. We prove that this is indeed the case for model M1. We start with a simple lemma:

Lemma 5 Let $\sigma$ and $\tilde{\sigma}$ be nonnegative, with $\sigma \preceq$ st $\tilde{\sigma}$. Let $\varepsilon$ be independent from $\sigma$ and $\tilde{\sigma}$, with $E[\varepsilon]=0$; then $\sigma \varepsilon \preceq_{c x} \tilde{\sigma} \varepsilon$.

Proof. We can construct identically distributed copies of $\sigma$ and $\tilde{\sigma}$ on the same probability space, such that $\sigma \leq \tilde{\sigma}$ a.s. Then for each realization of $\sigma$, we have that $\sigma \varepsilon \preceq_{c x} \tilde{\sigma} \varepsilon$. From Theorem 3.A.12 in [13] it follows that $\sigma \varepsilon \preceq_{c x} \tilde{\sigma} \varepsilon$.

\section{Theorem 6 Propagation of convex order}

Let $X_{n}$ be as in (11) and $\varepsilon_{k} \preceq_{c x} \widetilde{\varepsilon}_{k}$; it follows that $X_{n} \preceq_{c x} \widetilde{X}_{n}$

Proof. First of all we remark that since $\varepsilon_{k} \preceq_{c x} \widetilde{\varepsilon}_{k}$, it follows that $\left|\varepsilon_{k}\right| \preceq_{i c x}$ $\left|\widetilde{\varepsilon}_{k}\right|$. Indeed, for each $\phi$ increasing and convex, the composition $\phi(|\ldots|)$ is convex; this implies that $E\left[\phi\left(\left|\varepsilon_{k}\right|\right)\right] \leq E\left[\phi\left(\left|\widetilde{\varepsilon}_{k}\right|\right)\right]$, that is $\left|\varepsilon_{k}\right| \preceq_{i c x}\left|\widetilde{\varepsilon}_{k}\right|$. From Proposition 3 item b), it then follows that $\sigma_{n+1} \preceq_{i c x} \widetilde{\sigma}_{n+1}$. From Theorem 4.A.6 in [13] there exists a random variable $\bar{\sigma}_{n+1}$ with $\sigma_{n+1} \preceq_{s t} \bar{\sigma}_{n+1} \preceq_{c x} \widetilde{\sigma}_{n+1}$. By Lemma 5 , we have that $\sigma_{n+1} \preceq_{s t} \bar{\sigma}_{n+1}$ implies that $\sigma_{n+1} \varepsilon_{n+1} \preceq_{c x} \bar{\sigma}_{n+1} \varepsilon_{n+1}$; on the other hand $\bar{\sigma}_{n+1} \preceq_{c x} \widetilde{\sigma}_{n+1}$ implies that $\bar{\sigma}_{n+1} \varepsilon_{n+1} \preceq_{c x} \widetilde{\sigma}_{n+1} \varepsilon_{n+1}$. By transitivity we get $\sigma_{n+1} \varepsilon_{n+1} \preceq_{c x} \widetilde{\sigma}_{n+1} \varepsilon_{n+1}$.

\section{The relevant orderings}

In the preceding section the orderings defined by $|X| \preceq_{s t}|Y|, X^{2} \preceq_{s t} Y^{2}$, $|X| \preceq_{i c x}|Y|, X^{2} \preceq_{i c x} Y^{2}$ have arisen naturally.

In order to better understand their meaning, in the following lemmas we identify some necessary and sufficient conditions in the continuous and symmetric case. We have the following:

Lemma 7 Let $X$ and $Y$ be symmetric with continuous distributions $F$ and $G$. The following conditions are equivalent:

a) $X^{2} \preceq_{s t} Y^{2}$;

b) $|X| \preceq_{s t}|Y|$;

c) $X \preceq_{\text {peak }} Y$, where $\preceq_{\text {peak }}$ is the peakedness ordering introduced in [3];

d) $S^{-}(G-F)=1$ with sign sequence,+- , where $S^{-}(G-F)$ is the number of intersections between $G$ and $F$ as defined in (1.A.18) of [13].

Proof. The equivalence of a) and b) is an immediate consequence of Theorem 1.A.3 in [13]. The equivalence of b) and c) is the definition of the peakedness 
ordering, while the equivalence between c) and d) follows from Theorem 3.D.1 in 13 .

Lemma 8 Let $X$ and $Y$ be symmetric with continuous distributions $F$ and $G$. The following conditions are equivalent:

a) $X^{2} \preceq_{i c x} Y^{2}$

b) $\int_{x}^{+\infty} \bar{F}(u) \cdot u \cdot d u \leq \int_{x}^{+\infty} \bar{G}(u) \cdot u \cdot d u$ for each $x \geq 0$, where $\bar{F}(u)=1-F(u)$ and $\bar{G}(u)=1-G(u)$

c) $E\left[\left(X^{2}-k\right)^{+}\right] \leq E\left[\left(Y^{2}-k\right)^{+}\right]$for each $k \geq 0$

Proof. Under our hypothesis $F_{X^{2}}(t)=2 F(\sqrt{t})-1$ and $\bar{F}_{X^{2}}(t)=2-2 F(\sqrt{t})$, for $t \geq 0$. The equivalence of a) and b) follows then from Theorem 4.A.2 in [13] with a simple change of variable. The equivalence between a) and c) is also a consequence of Theorem 1.5.7 in [10]

The first lemma shows that for symmetric variables the orderings $|X| \preceq$ st $|Y|$ and $X^{2} \preceq_{s t} Y^{2}$ are variability comparisons equivalent to the peakedness ordering, that in this case boils down to item $\mathrm{d}$ ), that is the validity of a single cut condition between the distribution functions. In the typical econometric applications these orderings are however not very relevant since the innovations satisfy $E\left[\varepsilon_{k}^{2}\right]=1$, and hence $E\left[\varepsilon_{k}^{2}\right] \preceq s t E\left[\widetilde{\varepsilon}_{k}^{2}\right]$ would imply $\varepsilon_{k}^{2}={ }_{s t} \widetilde{\varepsilon}_{k}^{2}$.

In the normalized case the ordering $X^{2} \preceq_{i c x} Y^{2}$ becomes equivalent to $X^{2} \preceq_{c x} Y^{2}$; we prove a sufficient and a necessary condition for it.

Lemma 9 Let $X$ and $Y$ be symmetric with continuous distributions $F$ and $G$ and with $E\left[X^{2}\right]=E\left[Y^{2}\right]=1$.

a) If the densities of $X$ and $Y$ cross 4 times, with the density of $X$ being lower in the tails and in the center, and higher in the intermediate region, then $X^{2} \preceq_{i c x}$ $Y^{2}$.

b) If $X^{2} \preceq_{i c x} Y^{2}$ and $X$ and $Y$ have finite fourth moments, then $\beta_{2}(X)<\beta_{2}(Y)$, where $\beta_{2}$ is Pearson's kurtosis coefficient.

Proof. a) Under our hypothesis $f_{X^{2}}(t)=\frac{f(\sqrt{t})}{\sqrt{t}}$ for $t>0$. Since $X$ and $Y$ are symmetrical, we have that the four intersection points between the densities $f$ and $g$ are symmetrical with respect to the origin. Hence the densities of $X^{2}$ and $Y^{2}$ cross in two points and since $E\left[X^{2}\right]=E\left[Y^{2}\right]$ from Theorem 3.A.44 in [13] we have that $X^{2} \preceq_{c x} Y^{2}$.

b) In our case $\beta_{2}(X)=E\left[X^{4}\right]$ and hence the thesis follows from the definition of the convex order.

This lemma shows that the comparison $X^{2} \preceq_{i c x} Y^{2}$ can be interpreted as a classical kurtosis ordering; the cut condition is usually referred in the kurtosis ordering literature as a Dyson-Finucan condition (see for example [7] for a review). 


\section{Convex comparisons for total logreturns}

In financial applications the variables $X_{n}$ typically represent logreturns, that are additive quantities. The over-the-period total return is given by $S_{n}=\sum_{k=1}^{n} X_{n}$. It is therefore natural to ask if some of the comparison results of Section 2 do extend to the variables $S_{n}$. In this section we consider the case of the convex order, that is, we wonder if $\varepsilon_{k} \preceq_{c x} \widetilde{\varepsilon}_{k} \Rightarrow S_{n} \preceq_{c x} \widetilde{S}_{n}$. The problem is not trivial since $S_{n}$ cannot be expressed as a sum of independent variables, so the standard results about convex ordering of sums cannot be applied; we are able to prove a positive result in the case of model M1 and for symmetric innovations. We start with a basic lemma:

Lemma 10 Let $\phi \in C^{2}(\mathbb{R})$ be convex and $g_{i} \in C^{2}(\mathbb{R})$ be convex and nonnegative. Let $a, b \in R$ and $P_{m}:=\{-1,1\}^{m}$. It follows that

$$
h(u)=\sum_{\underline{p} \in P_{m}} \phi\left(a+b u+\sum_{i=1}^{m} p_{i} g_{i}(u)\right)
$$

is convex.

Proof. We can compute

$$
\begin{aligned}
h^{\prime}(u)= & \sum_{\underline{p} \in P_{m}} \phi^{\prime}\left(a+b u+\sum_{i=1}^{m} p_{i} g_{i}(u)\right) \cdot\left(b+\sum_{i=1}^{m} p_{i} g_{i}^{\prime}(u)\right) \\
h^{\prime \prime}(u)= & \sum_{\underline{p} \in P_{m}} \phi^{\prime \prime}\left(a+b u+\sum_{i=1}^{m} p_{i} g_{i}(u)\right) \cdot\left(b+\sum_{i=1}^{m} p_{i} g_{i}^{\prime}(u)\right)^{2}+ \\
& \sum_{\underline{p} \in P_{m}} \phi^{\prime}\left(a+b u+\sum_{i=1}^{m} p_{i} g_{i}(u)\right) \cdot\left(\sum_{i=1}^{m} p_{i} g_{i}^{\prime \prime}(u)\right)
\end{aligned}
$$

The first term is positive; the second is given by

$$
A_{m}=\sum_{\underline{p} \in P_{m}} \phi^{\prime}\left(a+b u+\sum_{i=1}^{m} p_{i} g_{i}(u)\right) \cdot\left(\sum_{i=1}^{m} p_{i} g_{i}^{\prime \prime}(u)\right)
$$

Let us denote with $\underline{P}$ a random vector with a discrete uniform distribution on $P_{m}$; clearly $E[\underline{P}]=\underline{0}$, the components of $\underline{P}$ are independent and

$$
A_{m}=2^{m} E\left[\phi^{\prime}(a+b u+\underline{g}(u) \cdot \underline{P})\left(\underline{g}^{\prime \prime}(u) \cdot \underline{P}\right)\right]
$$

Since the functions $\phi^{\prime}(a+b u+\underline{g}(u) \cdot \underline{P})$ and $\underline{g}^{\prime \prime}(u) \cdot \underline{P}$ are componentwise increasing, from the covariance inequality it follows that

$$
\begin{aligned}
A_{m} & =2^{m} E\left[\phi^{\prime}(\underline{g}(u) \cdot \underline{P})\left(\underline{g}^{\prime \prime}(u) \cdot \underline{P}\right)\right] \geq \\
& \geq 2^{m} E\left[\phi^{\prime}(\underline{g}(u) \cdot \underline{P})\right] E\left[\left(\underline{g}^{\prime \prime}(u) \cdot \underline{P}\right)\right]=0
\end{aligned}
$$


We remark that in this lemma the smoothness requirements on $\phi$ and on the $g_{i}$ can be dropped; we preferred this formulation in order to simplify the proof. Since in this section we consider only model M1, we define

$$
g_{n}\left(\sigma_{0}, \varepsilon_{0}, \ldots, \varepsilon_{n-1}\right):=g_{n}^{I}\left(\sigma_{0},\left|\varepsilon_{0}\right|, \ldots,\left|\varepsilon_{n-1}\right|\right) ;
$$

from Lemma 1, it is clear that $g_{n}$ is even and ccx. We have

$$
\begin{aligned}
S_{n}= & X_{0}+X_{1}+\ldots+X_{n}=\sigma_{0} \varepsilon_{0}+\sigma_{1} \varepsilon_{1}+\ldots+\sigma_{n} \varepsilon_{n}= \\
& \sigma_{0} \varepsilon_{0}+g_{1}\left(\sigma_{0}, \varepsilon_{0}\right) \varepsilon_{1}+\ldots+g_{n}\left(\sigma_{0}, \varepsilon_{0}, \ldots, \varepsilon_{n-1}\right) \varepsilon_{n}= \\
& S_{n}\left(\sigma_{0}, \varepsilon_{0}, \ldots, \varepsilon_{n}\right)
\end{aligned}
$$

The main problem in proving the propagation of convexity to the sums is that $S_{n}$ is not a ccx functions of the innovations $\varepsilon_{k}$; indeed, each $g_{k}$ in (5) is multiplied by a possibly negative innovation $\varepsilon_{k}$. This prevents the applications of standard results and requires the development of a specific technique based on Lemma 10. The basic idea is that in the case of symmetric innovations it is possible to restore the convexity by averaging over all the possible sign changes, as in Lemma 10. This will be done in a recursive way; we start with the following:

Lemma 11 Let $X_{n}$ and $S_{n}$ be as in (11) and (5). Let $\phi$ be convex and $\varepsilon_{i}$ be symmetric. Then the function

$$
h\left(\sigma_{0}, \varepsilon_{0}, \ldots, \varepsilon_{k}\right):=E_{\varepsilon_{k+1}, \ldots, \varepsilon_{n}}\left[\phi\left(S_{n}\left(\sigma_{0}, \varepsilon_{0}, \ldots, \varepsilon_{n}\right)\right)\right]
$$

is convex in $\varepsilon_{k}$ for each fixed value of $\sigma_{0}, \varepsilon_{0}, \ldots, \varepsilon_{k-1}$.

Proof. To avoid notational burdening we drop the arguments of the functions $g_{i}$. Since the innovations are symmetric and $g_{i}$ is even, we can write

$$
\begin{gathered}
E_{\varepsilon_{k+1}, \ldots, \varepsilon_{n}}\left[\phi\left(S_{n}\left(\sigma_{0}, \varepsilon_{0}, \ldots, \varepsilon_{n}\right)\right)\right]=E_{\varepsilon_{k+1}, \ldots, \varepsilon_{n}}\left[\phi\left(\sigma_{0} \varepsilon_{0}+\ldots+g_{n} \varepsilon_{n}\right)\right]= \\
E_{\varepsilon_{k+1}, \ldots, \varepsilon_{n}}\left[\sum_{\underline{p} \in P_{n-k}} \phi\left(\sigma_{0} \varepsilon_{0}+\ldots+p_{1} g_{k+1} \varepsilon_{k+1}+\ldots+p_{n-k} g_{n} \varepsilon_{n}\right) 1_{\left\{\varepsilon_{k+1} \geq 0, \ldots, \varepsilon_{n} \geq 0\right\}}\right] .
\end{gathered}
$$

Denoting by

$\bar{h}\left(\sigma_{0}, \varepsilon_{0}, \ldots, \varepsilon_{k}, \ldots, \varepsilon_{n}\right)=\sum_{\underline{p} \in P_{n-k}} \phi\left(\sigma_{0} \varepsilon_{0}+g_{1} \varepsilon_{1}+\ldots+g_{k} \varepsilon_{k}+p_{1} g_{k+1} \varepsilon_{k+1}+\ldots+p_{n-k} g_{n} \varepsilon_{n}\right)$,

we have that

$$
h\left(\sigma_{0}, \varepsilon_{0}, \ldots, \varepsilon_{k-1}, \varepsilon_{k}\right)=E_{\varepsilon_{k+1}, \ldots, \varepsilon_{n}}\left[1_{\left\{\varepsilon_{k+1} \geq 0, \ldots, \varepsilon_{n} \geq 0\right\}} \bar{h}\left(\sigma_{0}, \varepsilon_{0}, \ldots, \varepsilon_{k}, \ldots, \varepsilon_{n}\right)\right]
$$

and $\bar{h}$ is convex in $\varepsilon_{k}$ from Lemma 10 It follows that also $h\left(\sigma_{0}, \varepsilon_{0}, \ldots, \varepsilon_{k-1}, \varepsilon_{k}\right)$ is convex in $\varepsilon_{k}$ for each value of $\sigma_{0}, \varepsilon_{0}, \ldots, \varepsilon_{k-1}$.

We can finally state the result on the propagation of the convex order to $S_{n}$ : 
Theorem 12 Let $X_{n}$ and $S_{n}$ be as in (11) and (5). Let $\varepsilon_{i}$ be symmetric. If also $\widetilde{\varepsilon}_{k}$ is symmetric and $\widetilde{\varepsilon}_{k} \geq_{c x} \varepsilon_{k}$, then $\widetilde{S}_{n}:=S_{n}\left(\sigma_{0}, \varepsilon_{0}, . . \widetilde{\varepsilon}_{k}, \ldots, \varepsilon_{n}\right) \geq_{c x}$ $S_{n}\left(\sigma_{0}, \varepsilon_{0}, ., \varepsilon_{k}, \ldots, \varepsilon_{n}\right)$.

Proof. Let $\phi$ be convex. From the independence of the $\varepsilon_{i}$ we can write

$$
\begin{aligned}
E\left[\phi\left(\widetilde{S}_{n}\right)\right] & =E_{\varepsilon_{0}, \ldots, \varepsilon_{k-1}} E_{\widetilde{\varepsilon}_{k}} E_{\varepsilon_{k+1}, \ldots, \varepsilon_{n}}\left[\phi\left(S_{n}\left(\sigma_{0}, \varepsilon_{0}, \ldots, \varepsilon_{k-1}, \widetilde{\varepsilon}_{k}, \varepsilon_{k+1}, \ldots, \varepsilon_{n}\right)\right)\right]= \\
& =E_{\varepsilon_{0}, \ldots, \varepsilon_{k-1}} E_{\widetilde{\varepsilon}_{k}}\left[h\left(\sigma_{0}, \varepsilon_{0}, \ldots, \varepsilon_{k-1}, \widetilde{\varepsilon}_{k}\right)\right]
\end{aligned}
$$

where as in (6)

$$
h\left(\sigma_{0}, \varepsilon_{0}, \ldots, \varepsilon_{k-1}, \widetilde{\varepsilon}_{k}\right):=E_{\varepsilon_{k+1}, \ldots, \varepsilon_{n}}\left[\phi\left(S_{n}\left(\sigma_{0}, \varepsilon_{0}, \ldots, \varepsilon_{k-1}, \widetilde{\varepsilon}_{k}, \varepsilon_{k+1}, \ldots, \varepsilon_{n}\right)\right)\right]
$$

is a convex function of $\widetilde{\varepsilon}_{k}$ for each value of $\sigma_{0}, \varepsilon_{0}, \ldots, \varepsilon_{k-1}$ from Lemma 11 It follows that

$$
E_{\widetilde{\varepsilon}_{k}}\left[h\left(\sigma_{0}, \varepsilon_{0}, \ldots, \varepsilon_{k-1}, \widetilde{\varepsilon}_{k}\right)\right] \geq E_{\varepsilon_{k}}\left[h\left(\sigma_{0}, \varepsilon_{0}, \ldots, \varepsilon_{k-1}, \varepsilon_{k}\right)\right]
$$

that gives

$$
\begin{aligned}
E\left[\phi\left(\widetilde{S}_{n}\right)\right] & =E_{\varepsilon_{0}, \ldots, \varepsilon_{k-1}} E_{\widetilde{\varepsilon}_{k}}\left[h\left(\sigma_{0}, \varepsilon_{0}, \ldots, \varepsilon_{k-1}, \widetilde{\varepsilon}_{k}\right)\right] \geq \\
& \geq E_{\varepsilon_{0}, \ldots, \varepsilon_{k-1}} E_{\varepsilon_{k}}\left[h\left(\sigma_{0}, \varepsilon_{0}, \ldots, \varepsilon_{k-1}, \varepsilon_{k}\right)\right]=E\left[\phi\left(S_{n}\right)\right]
\end{aligned}
$$

that is $\widetilde{S}_{n} \geq_{c x} S_{n}$.

\section{Multivariate comparisons of logreturns}

Until now we have been considering only univariate orderings of the $X_{n}$. Having established also a convex comparison result for the sums $S_{n}$, it is natural to wonder whether more general multivariate comparisons do hold, that is if we can prove that $\varepsilon_{k} \leq_{c x} \widetilde{\varepsilon}_{k} \Rightarrow\left(X_{1}, \ldots, X_{n}\right) \preceq\left(\widetilde{X}_{1}, \ldots, \widetilde{X}_{n}\right)$ for some multivariate convexity ordering $\preceq$ to be precise in the sequel. Before stating a positive result, we recall two basic definitions:

Definition 13 A function $\varphi: \mathbb{R}^{n} \rightarrow \mathbb{R}$ is directionally convex if for any $\mathbf{x}_{1}, \ldots, \mathbf{x}_{4} \in \mathbb{R}^{n}$, such that $\mathbf{x}_{1} \leq \mathbf{x}_{2}, \mathbf{x}_{3} \leq \mathbf{x}_{4}$ and $\mathbf{x}_{1}+\mathbf{x}_{4}=\mathbf{x}_{2}+\mathbf{x}_{3}$, it holds that

$$
\varphi\left(\mathbf{x}_{2}\right)+\varphi\left(\mathbf{x}_{3}\right) \leq \varphi\left(\mathbf{x}_{1}\right)+\varphi\left(\mathbf{x}_{4}\right)
$$

Definition 14 A function $\varphi: \mathbb{R}^{n} \rightarrow \mathbb{R}$ is supermodular if for any $\mathbf{x}, \mathbf{y} \in \mathbb{R}^{n}$ it satisfies:

$$
\varphi(\mathbf{x})+\varphi(\mathbf{y}) \leq \varphi(\mathbf{x} \wedge \mathbf{y})+\varphi(\mathbf{x} \vee \mathbf{y})
$$

where the operators $\wedge$ and $\vee$ denote respectively coordinatewise minimum and maximum (see Section 7.A.8 of [13]) 
In the univariate case directionally convexity is equivalent to convexity, while in the multivariate case there are no implications between the two concepts. Directionally convexity implies supermodularity and its equivalent to supermodularity plus componentwise convexity. For smooth functions, directionally convexity is equivalent to the nonnegativity of all entries in the Hessian matrix, while supermodularity is equivalent to the the nonnegativity of all entries out of the principal diagonal. Clearly no implications exist between this concept and the usual convexity of $\phi$, that corresponds to the positive semidefiniteness of the Hessian matrix. However, $\phi$ is directionally convex and convex if and only if it is supermodular and convex. Finally, in the smooth case, $\varphi$ is directionally convex if and only if its gradient is increasing.

In order to establish multivariate comparison results, we need a generalization of Lemma 10 .

Lemma 15 Let $\phi \in C^{2}\left(\mathbb{R}^{n}\right)$ be convex and supermodular and $g_{i} \in C^{2}(\mathbb{R})$ be convex and nonnegative. Let $P_{n}:=\{-1,1\}^{n}$. It follows that

$$
h(u)=\sum_{\underline{p} \in P_{n}} \phi\left(p_{1} g_{1}(u), \ldots, p_{n} g_{n}(u)\right)
$$

is convex.

Proof. Let's denote by $y_{i}$ the arguments of the function $\phi$; we can write:

$$
\begin{gathered}
h^{\prime}(u)=\sum_{\underline{p} \in P_{m}} \sum_{i=1}^{m} p_{i} g_{i}^{\prime}(u) \frac{\partial \phi}{\partial y_{i}}\left(p_{1} g_{1}(u), \ldots, p_{m} g_{m}(u)\right) \\
h^{\prime \prime}(u)=\sum_{\underline{p} \in P_{m}}\left[\sum_{i=1}^{m} p_{i} g_{i}^{\prime \prime}(u) \frac{\partial \phi}{\partial y_{i}}\left(p_{1} g_{1}(u), \ldots, p_{m} g_{m}(u)\right)+\right. \\
\left.+\sum_{i=1}^{m} \sum_{j=1}^{m} p_{i} p_{j} g_{i}^{\prime}(u) g_{j}^{\prime}(u) \frac{\partial^{2} \phi}{\partial y_{i} \partial y_{j}}\left(p_{1} g_{1}(u), \ldots, p_{m} g_{m}(u)\right)\right]
\end{gathered}
$$

The second term in the square brackets can be written as

$\left.\sum_{i=1}^{m} \sum_{j=1}^{m} p_{i} p_{j} g_{i}^{\prime}(u) g_{j}^{\prime}(u) \frac{\partial^{2} \phi}{\partial y_{i} \partial y_{j}}\left(p_{1} g_{1}(u), \ldots, p_{m} g_{m}(u)\right)=\underline{P g^{\prime}}(u)\left[\frac{\partial^{2} \phi}{\partial y_{i} \partial y_{j}}(.).\right] \underline{\left(P g^{\prime}\right.}(u)\right)^{T} \geq 0$

and is positive since the Hessian of $\phi$ is positive semidefinite.

The first term can be written as

$$
\sum_{\underline{p} \in P_{m}} \sum_{i=1}^{m} p_{i} g_{i}^{\prime \prime}(u) \frac{\partial \phi}{\partial y_{i}}\left(p_{1} g_{1}(u), \ldots, p_{m} g_{m}(u)\right)=2^{m} E\left[\nabla \phi\left(p_{1} g_{1}(u), \ldots, p_{n} g_{n}(u)\right) \cdot\left(\underline{P g}^{\prime \prime}(u)\right)\right]
$$


From the hypothesis on $\phi$ all the components of $\nabla \phi$ are increasing in $p_{i}$, hence from the multivariate covariance inequality

$$
E\left[\nabla \phi\left(p_{1} g_{1}(u), \ldots, p_{n} g_{n}(u)\right) \cdot\left(\underline{P g}^{\prime \prime}(u)\right)\right] \geq 0
$$

As in Lemma 10, the smoothness requirements on $\phi$ and $g_{i}$ can be dropped, but we added them in order to simplify the proof. The multivariate analogue of Lemma 11 is the following:

Lemma 16 Let $X_{n}$ and $S_{n}$ be as in (1) and (5). Let $\phi: R^{n+1} \rightarrow R$ be supermodular and convex and $\varepsilon_{i}$ be symmetric. Then the function

$$
h_{k}(x):=E\left[\phi\left(X_{0}, \ldots, X_{n}\right) \mid \varepsilon_{k}=x\right] ;
$$

is convex.

Proof. From the symmetry of the innovations we can write

$$
\begin{gathered}
h_{k}(x)=E_{\varepsilon_{0} \ldots \varepsilon_{k-1} \varepsilon_{k+1} \ldots \varepsilon_{n}}\left[\phi\left(\sigma_{0} \varepsilon_{0}, g_{1} \varepsilon_{1}, \ldots, g_{k} x,, \ldots, g_{n} \varepsilon_{n}\right)\right]= \\
=E_{\varepsilon_{0} \ldots \varepsilon_{k-1} \varepsilon_{k+1} \ldots \varepsilon_{n}}\left[1_{\{\underline{\varepsilon} \geq \underline{0}\}} \sum_{\underline{p} \in P_{n}} \phi\left(\sigma_{0} p_{0} \varepsilon_{0}, g_{1} p_{1} \varepsilon_{1}, \ldots, g_{k} p_{k} x, \ldots, g_{n} p_{n} \varepsilon_{n}\right)\right]
\end{gathered}
$$

Since each $g_{i}$ is convex in $\varepsilon_{k}$, from Lemma 15] it follows that for each $\sigma_{0}>0$ and $\varepsilon_{i} \geq 0, i \neq k$, the function

$$
\bar{h}_{k}(x)=\sum_{\underline{p} \in P_{n}} \phi\left(\sigma_{0} p_{0} \varepsilon_{0}, g_{1} p_{1} \varepsilon_{1}, \ldots, g_{k} p_{k} x, \ldots, g_{n} p_{n} \varepsilon_{n}\right)
$$

is convex. Averaging with respect to $\varepsilon_{i}$, with $i \neq k$, it follows that also $h_{k}(x)$ is convex.

This enables us to state our main multivariate comparison result:

Theorem 17 Let $X_{n}$ and $S_{n}$ be as in (1) and (5)). Let the $\varepsilon_{i}$ be symmetric. If also $\widetilde{\varepsilon}_{k}$ is symmetric and $\widetilde{\varepsilon}_{k} \geq_{c x} \varepsilon_{k}$, then,

$$
E\left[\phi\left(X_{0}, \ldots, X_{k}, \ldots, X_{n}\right)\right] \leq E\left[\phi\left(X_{0}, \ldots, \tilde{X}_{k}, \ldots, \tilde{X}_{n}\right)\right]
$$

for every function $\phi: R^{n+1} \rightarrow R$ supermodular and convex.

Proof. From the previous lemma we have that

$$
E\left[\phi\left(X_{0}, \ldots, X_{k}, \ldots, X_{n}\right)\right]=E_{\varepsilon_{k}}\left[h_{k}\left(\varepsilon_{k}\right)\right] \leq E_{\widetilde{\varepsilon}_{k}}\left[h_{k}\left(\widetilde{\varepsilon}_{k}\right)\right]=E\left[\phi\left(X_{0}, \ldots \tilde{X}_{k}, \ldots, \tilde{X}_{n}\right)\right] .
$$

We remark that we are not able to prove supermodularity or componentwise convex ordering of $\left(X_{0}, \ldots, X_{n}\right)$; at the moment both hypothesys on $\phi$ seem to be necessary for Lemma 15 . 


\section{The Garch $(1,1)$ case}

We focus now on the Garch $(1,1)$ model specified by

$$
\left\{\begin{array}{c}
X_{n}=\sigma_{n} \varepsilon_{n} \\
\sigma_{n+1}^{2}=\alpha_{0}+\alpha_{1} X_{n}^{2}+\beta_{1} \sigma_{n}^{2}
\end{array},\right.
$$

with $\alpha_{0}, \alpha_{1}, \beta_{1}>0$ and $\alpha_{1}+\beta_{1}<1$. For this model the recursive dynamic of the volatility or of the variance (4) can easily be explicitated as follows (see [12]):

$$
\sigma_{n+1}^{2}=\sigma_{0}^{2} \prod_{i=1}^{n+1}\left(\beta_{1}+\alpha_{1} \varepsilon_{n-i+1}^{2}\right)+\alpha_{0}\left[1+\sum_{k=1}^{n} \prod_{i=1}^{k}\left(\beta_{1}+\alpha_{1} \varepsilon_{n-i+1}^{2}\right)\right]
$$

From this expression it is immediate that $\sigma_{n+1}^{2}$ and $\sigma_{n+1}$ are nondecreasing functions of the parameters $\alpha_{0}, \alpha_{1}$ and $\beta_{1}$. We already remarked that this model is a special case of both M1 and M2, so all the comparison result for varying innovations of the preceding sections do hold. In this section we are interested in establishing comparison results for different parameters $\alpha_{0}, \alpha_{1}, \beta_{1}$. As mentioned in the Introduction, it is natural that an increase in $\alpha_{0}, \alpha_{1}, \beta_{1}$ should correspond to an increase in the variability of $X_{n}$ and $S_{n}$; in this section we prove it rigorously. Without any additional effort, we can consider stochastic parameters $\alpha_{0}, \alpha_{1}, \beta_{1}$ :

Proposition 18 Let $X_{n}$ be as in (7). If we consider random parameters $\alpha_{0} \leq_{s t}$ $\widetilde{\alpha}_{0}, \alpha_{1} \leq_{s t} \widetilde{\alpha}_{1}$ and $\beta_{1} \leq_{s t} \widetilde{\beta}_{1}$, then $\left|X_{n}\right| \leq_{s t}\left|\widetilde{X}_{n}\right|, X_{n}^{2} \leq_{s t} \widetilde{X}_{n}^{2}$ and $X_{n} \preceq_{c x} \widetilde{X}_{n}$.

Proof. Since $\sigma_{n}$ and $\sigma_{n}^{2}$ are increasing functions of the parameters, if $\alpha_{0} \leq_{s t}$ $\widetilde{\alpha}_{0}, \alpha_{1} \leq_{s t} \widetilde{\alpha}_{1}$ and $\beta_{1} \leq_{s t} \widetilde{\beta}_{1}$ it follows that $\sigma_{n} \leq_{s t} \widetilde{\sigma}_{n}$ and $\sigma_{n}^{2} \leq_{s t} \widetilde{\sigma}_{n}^{2}$. As in the proof of Theorem 4 it follows that $\left|X_{n}\right| \leq_{s t}\left|\widetilde{X}_{n}\right|$ and $X_{n}^{2} \leq_{s t} \tilde{X}_{n}^{2}$. From Lemma $5 \sigma_{n} \leq_{s t} \widetilde{\sigma}_{n}$ implies that $X_{n} \preceq_{c x} \widetilde{X}_{n}$.

The last point is to prove the convex comparison of the sums $S_{n}$; again, this is nontrivial since the $X_{n}$ are not independent; we provide a proof in the case of symmetric innovations.

Theorem 19 Let $X_{n}$ be as in (7) and $S_{n}$ as in (5). Let $\varepsilon_{i}$ be symmetric. If we consider random parameters $\alpha_{0} \leq_{s t} \widetilde{\alpha}_{0}, \alpha_{1} \leq_{s t} \widetilde{\alpha}_{1}$ and $\beta_{1} \leq_{s t} \widetilde{\beta}_{1}$, then $S_{n} \preceq_{c x} \tilde{S}_{n}$.

Proof. As before, we write

$$
S_{n}=\sigma_{0} \varepsilon_{0}+g_{1}\left(\varepsilon_{0}, \ldots, \alpha_{0}, \alpha_{1}, \beta_{1}\right) \varepsilon_{1}+\ldots+g_{n}\left(\varepsilon_{0}, \ldots, \alpha_{0}, \alpha_{1}, \beta_{1}\right) \varepsilon_{n}
$$

where the functions $g_{i}$ are nondecreasing in the parameters $\alpha_{0}, \alpha_{1}, \beta_{1}$. Let $\phi$ be any convex function. We first want to prove that $E\left[\phi\left(S_{n}\right)\right]$ is nondecreasing 
in the parameters $\alpha_{0}, \alpha_{1}, \beta_{1}$. From the symmetry of the innovations $\varepsilon_{i}$ we can write:

$$
\begin{gathered}
E\left[\phi\left(S_{n}\right)\right]=E_{\varepsilon_{0}, \ldots, \varepsilon_{n}}\left[\phi\left(\sigma_{0} \varepsilon_{0}+\ldots+g_{n} \varepsilon_{n}\right)\right] \\
=E_{\varepsilon_{0}, \ldots, \varepsilon_{n}}\left[\sum_{\underline{p} \in P_{n+1}} \phi\left(\sigma_{0} p_{0} \varepsilon_{0}+\ldots+p_{n} g_{n} \varepsilon_{n}\right) 1_{\left\{\varepsilon_{0} \geq 0, \ldots, \varepsilon_{n} \geq 0\right\}}\right] .
\end{gathered}
$$

Denoting by

$$
\bar{h}\left(\sigma_{0}, \ldots, \alpha_{0}, \alpha_{1}, \beta_{1}\right)=\sum_{\underline{p} \in P_{n+1}} \phi\left(\sigma_{0} p_{0} \varepsilon_{0}+\ldots+p_{n} g_{n} \varepsilon_{n}\right),
$$

we see that $\bar{h}$ is nondecreasing in $\alpha_{0}, \alpha_{1}, \beta_{1}$; indeed we can compute:

$$
\frac{\partial \bar{h}}{\partial \alpha_{0}}=\sum_{\underline{p} \in P_{n+1}} \phi^{\prime}\left(\sigma_{0} p_{0} \varepsilon_{0}+\ldots+p_{n} g_{n} \varepsilon_{n}\right) \cdot\left(p_{1} \varepsilon_{1} g_{1}^{\prime}+\ldots+p_{n} \varepsilon_{n} g_{n}^{\prime}\right) \geq 0
$$

from the multivariate covariance inequality, as in the proof of Lemma 10 . The same reasoning shows that $\frac{\partial \bar{h}}{\partial \alpha_{1}} \geq 0$ and $\frac{\partial \bar{h}}{\partial \beta_{1}} \geq 0$.

It follows that $E\left[\phi\left(S_{n}\right)\right]$ is is nondecreasing in $\alpha_{0}, \alpha_{1}, \beta_{1}$; but then if $\alpha_{0} \leq_{s t} \widetilde{\alpha}_{0}$, $\alpha_{1} \leq_{s t} \widetilde{\alpha}_{1}$ and $\beta_{1} \leq_{s t} \widetilde{\beta}_{1}$,

$$
E\left[\phi\left(S_{n}\left(\alpha_{0}, \alpha_{1}, \beta_{1}\right)\right] \leq E\left[\phi\left(S_{n}\left(\tilde{\alpha}_{0}, \tilde{\alpha}_{1}, \tilde{\beta}_{1}\right)\right]\right.\right.
$$

that is $S_{n} \preceq_{c x} \tilde{S}_{n}$.

\section{References}

[1] Bellamy, N., Jeanblanc, M. (2000). Incompleteness of markets driven by a mixed diffusion. Fin. Stochastics, 4, 209-222.

[2] Bergenthum, J., Rüschendorf, L. (2006). Comparison of option prices in semimartingale models. Fin. Stochastics, 10, 222-249.

[3] Birnbaum, Z. W. (1948). On random variables with comparable peakedness. Ann. Math. Stat., 19, 76-81.

[4] Bollerslev, T. (1986). General Autoregressive Conditional Heteroskedasticity. Journal of Econometrics, 31, 307-327.

[5] El Karoui, N., Jeanblanc-Picqué, M., Shreve, S. E. (1998). Robustness of the Black and Scholes formula. Math. Fin., 8, 93-126.

[6] Engle, R. (1982). Autoregressive Conditional Heteroskedasticity with Estimates of the Variance of the UK Inflation. Econometrica, 50, 987-1008.

[7] Finucan, H. M. (1964). A note on kurtosis. Journal of the Royal Statistical Society Series B (Methodological), 26, 1, 111-112. 
[8] Gushchin, A. A., Mordecki, E. (2002). Bounds on option prices for semi martingale market models. Proc. Steklov Inst. Math., 237, 73-113.

[9] Meester, L. E., Shanthikumar, J. G. (1999). Stochastic convexity on general space. Math. Oper. Res., 24, 472-494.

[10] Muller, A., Stoyan, D. (2002). Comparison Methods for Stochastic Models and Risks, J. Wiley \& Sons.

[11] Møller, T. (2004). Stochastic orders in dynamic insurance markets. Fin. Stochastics, 8, 479-499.

[12] Nelson, D.B. (1990). Stationarity and persistence in Garch(1,1) models. Econometric Theory, 6, 318-334.

[13] Shaked, M., Shantikumar, G. J. (2006). Stochastic Orders, 2nd edn., Springer. 